\title{
Exact accidental U(1) symmetries for the axion
}

\author{
Luc Darmé* and Enrico Nardi $\oplus^{\dagger}$ \\ INFN, Laboratori Nazionali di Frascati, C.P. 13, 100044 Frascati, Italy
}

(Received 24 February 2021; accepted 27 June 2021; published 13 September 2021)

\begin{abstract}
We study a class of gauge groups that can automatically yield a perturbatively exact Peccei-Quinn symmetry, and we outline a model in which the axion quality problem is solved at all operator dimensions. Gauge groups belonging to this class can also enforce and protect accidental symmetries of the clockwork type, and we present a toy model where an "invisible" axion arises from a single low scale breaking of the gauge and global symmetries.
\end{abstract}

DOI: 10.1103/PhysRevD.104.055013

\section{INTRODUCTION}

The nontrivial structure of the vacuum of Yang-Mills theories [1] implies that $C P$ violation is a built-in feature in QCD [2,3]. Strong $C P$ violation is parametrized in terms of an angular variable $\theta \in[0,2 \pi]$, whose value is not determined by the theory but is experimentally bounded to lie surprisingly close to zero $|\theta| \lesssim 10^{-10}$. It is hard to believe that this could simply occur as a whim of nature, especially because any value $\theta \lesssim 10^{-1}$ would leave our Universe basically unaffected [4-6], which precludes anthropic explanations. A convincing rationale for $\theta \approx 0$ is provided by the Peccei-Quinn (PQ) mechanism $[7,8]$, which postulates a global Abelian symmetry endowed with a mixed $U(1)_{\mathrm{PQ}}-S U(3)_{C}$ anomaly and broken spontaneously. This unavoidably entails a quasimassless spin zero boson, the axion $[9,10]$, whose central role is to relax dynamically $\theta$ to 0 . Remarkably, the axion also provides a novel solution to the origin of dark matter [11-13] as well as a plethora of other implications for astrophysics and cosmology (for a recent review, see [14]). However, it also raises various new issues. Among the deepest new questions stand the very origin of the axion or, more precisely, "which is the origin of the PQ symmetry"? There are in fact good reasons to believe that global symmetries cannot be fundamental, and this is especially true for anomalous symmetries that do not survive at the quantum level. A satisfactory explanation would arise if the PQ symmetry is enforced accidentally, in the sense that all renormalizable Lagrangian terms respecting first principles (Lorentz and local gauge invariance)

\footnotetext{
*luc.darme@lnf.infn.it

†enrico.nardi@lnf.infn.it
}

Published by the American Physical Society under the terms of the Creative Commons Attribution 4.0 International license. Further distribution of this work must maintain attribution to the author(s) and the published article's title, journal citation, and DOI. Funded by SCOAP ${ }^{3}$. automatically also preserve a global $U(1)$ with the required properties. A second issue, known as "the PQ symmetry quality problem," arises because to comply with the bound $|\theta|<10^{-10}, U(1)_{\mathrm{PQ}}$ must be respected by all effective operators acquiring a vacuum expectation value (VEV) up to dimension $D \gtrsim 11$. However, there is widespread consensus that global symmetries are violated by operators of all types and dimensions induced by quantum gravity effects [15-25]. A third issue regards the "the axion scale." The axion is a periodic field taking values in a compact space $a \in\left[0,2 \pi v_{a}\right]$, where the radius $v_{a}$ is generally identified with the PQ spontaneous symmetry breaking (SSB) scale $v_{\mathrm{PQ}}$. However, to comply with phenomenological constraints, $v_{a} \sim 10^{10 \pm 2} \mathrm{GeV}$ is required, and this brings in the usual problem of $\mathcal{O}\left(v_{\mathrm{PQ}}\right)$ corrections that can destabilize the electroweak scale.

Various strategies have been proposed to explain the origin of the PQ symmetry and to protect its quality up to a suitable operator dimension $D$ : discrete gauge symmetries $\mathbb{Z}_{D}$ [26-33], multiple complex scalars with values of $U(1)$ gauge charges of order $D$ [16], non-Abelian gauge symmetries generally of a degree not less than $D$ [34-37], often assisted by supersymmetry [38-40] or by higher dimensional constructions [41-44]. However, an unsatisfactory aspect of all these solutions is that if the scale of PQbreaking effects lies below the Planck scale, if SSB occurs at a scale $v_{\mathrm{PQ}} \gg 10^{10} \mathrm{GeV}$, or if future experimental limits will hint to $\theta \ll 10^{-10}$, the value of $D$ will have to be accordingly increased. With regards to the axion scale, certain solutions have been attempted, exploiting the socalled clockwork mechanism [45-54]. Clockwork PQ symmetries allow us to boost selectively some axion couplings [55-58], and to exponentially enhance [59-61] or suppress [62] the ratio $v_{a} / v_{\mathrm{PQ}}$. Clearly, the origin of clockwork symmetries also calls for an explanation, and in this case, achieving this goal by exploiting first principles is an even more challenging task. 
In this paper, we show that so far an uncharted class of flavor $^{1}$ gauge symmetries of the form $S U(M) \times S U(N)$ with $M \neq N$, that we denote as "rectangular" symmetries, allows us to solve at the root the origin and quality problems by enforcing automatically $U(1)$ symmetries that are either perturbatively exact at the Lagrangian level or that become exact on the vacuum. ${ }^{2}$ We outline a simple example where PQ protection is enforced by $S U(4) \times$ $S U(2)$. We also point out that rectangular symmetries can also prove useful to solve the axion scale problem. We illustrate this by constructing a toy model in which a clockwork $U(1)_{\mathrm{PQ}}$ arises automatically and is spontaneously broken together with the gauge symmetries by $v_{\mathrm{PQ}} \ll v_{a}$.

\section{RECTANGULAR GAUGE GROUPS AND ACCIDENTAL $U(1)_{P Q}$}

Consider a scalar multiplet $Y$ transforming in the bifundamental representation $(M, \bar{N})$ of the gauge group $\mathcal{G}_{M N}=S U(M) \times S U(N)$ with $M>N \geq 2$. Let us denote a generic component as $Y_{\alpha i}$, where greek indices span $S U(M)$ and latin indices $S U(N)$. Each group factor has a pair of Kronecker and Levi-Civita invariant tensors $\left(\delta_{M}, \epsilon_{M}\right),\left(\delta_{N}, \epsilon_{N}\right)$, which can be used to construct invariants by contracting the indices of field components. The renormalizable Lagrangian always contains $T \equiv \operatorname{Tr}\left(Y^{\dagger} Y\right)$ and $T_{4} \equiv \operatorname{Tr}\left(Y^{\dagger} Y\right)^{2}$ constructed from $\delta_{M}$ and $\delta_{N}$. Being Hermitian, they are manifestly invariant under a $U(1)_{\xi_{Y}}$ phase redefinition $Y \rightarrow e^{i \xi} Y$. Let us denote the trace of the matrix of the minors of $Y^{\dagger} Y$ of order $k$ as $C_{k}=$ $\operatorname{Tr}\left[\operatorname{Mnr}\left(Y^{\dagger} Y, k\right)\right]$. We have $C_{1} \equiv T, C_{2} \equiv A=\frac{1}{2}\left(T^{2}-T_{4}\right)$, and up to $C_{N}=\operatorname{det}\left[Y^{\dagger} Y\right]$ the $C_{k}$ 's form a fundamental set of $\mathcal{G}_{M N} \times U(1)_{\xi_{Y}}$ invariants $[64,65],{ }^{3}$ and it can be proven [64] that any higher order invariant $T_{2 k}=\operatorname{Tr}\left(Y^{\dagger} Y\right)^{k}$ can be expressed in terms of this set.

The accidental $U(1)_{\xi_{Y}}$ can only be broken by nonHermitian invariants, that is by operators with an unequal number of $Y$ and $Y^{\dagger}$ components, which must then involve the $\epsilon$ tensors. However, all invariants involving $\epsilon_{M}$ and a single scalar multiplet vanish symmetrically. Consider in fact the $S U(M)$ singlet,

$$
\epsilon^{\alpha_{1} \ldots \alpha_{M}} Y_{\alpha_{1}, i_{1}} \ldots Y_{\alpha_{M}, i_{M}} \equiv\left(\epsilon_{M} Y^{M}\right)_{i_{1} \ldots i_{M}},
$$

where the right-hand side defines a shorthand notation for $S U(M)$ indices contracted with $\epsilon_{M}$. Since $M>N$, at least

\footnotetext{
${ }^{1}$ In this work, "flavor" refers to a replication of exotic quarks.

${ }^{2}$ This term refers to global symmetries that are broken explicitly solely by operators whose VEV vanishes. Vacua having more global symmetries than the Lagrangian imply additional massless scalars besides the usual NambuGoldstone-Bosons (NGB) [63].

${ }^{3}$ Considering the $M \times M$ matrix $Y Y^{\dagger}$, one has $\operatorname{Tr}\left[\operatorname{Mnr}\left(Y Y^{\dagger}, k\right)\right]=C_{k}$ for $k \leq N$ and 0 for $k>N$, which yields the same result.
}

two components have the same $S U(N)$ index, and hence, the string vanishes. Thus, there are no invariants involving $\epsilon_{M}$ and a single scalar multiplet. ${ }^{4}$ Thus, the Lagrangian for a scalar multiplet $Y$ transforming under a rectangular gauge symmetry automatically enjoys a global $U(1)_{\xi_{Y}}$, which is perturbatively exact.

$U(1)_{\xi_{Y}}$ can be promoted to a PQ symmetry if it is endowed with a QCD anomaly. This requires assigning $U(1)_{\xi_{Y}}$ charges to fermions that carry color and couple to $Y$. Let us introduce two sets of chiral exotic quarks in the fundamental of $S U(3)_{C}$, singlets under the electroweak gauge group and transforming under $\mathcal{G}_{M N}$ as $\mathcal{Q}_{L} \sim(M, 1)$ and $Q_{R} \sim(1, N)$ so that the Yukawa operator $\overline{\mathcal{Q}}_{L} Y Q_{R}$ is gauge invariant. To prevent a gauge $S U(3)_{C}$ anomaly, we add $P=M-N$ quarks $q_{R}$ and a new scalar multiplet $Z$ acquiring a VEV so that all the quarks can be massive. This step can be arranged in different ways, the two extreme possibilities are

(I) Add a set of $\mathcal{G}_{M N}$-singlets $q_{R a}(a=1, \ldots, P)$, which couple to a scalar multiplet $Z \sim(M, 1)$ via $P$ Yukawa operators $\sum_{a=1}^{P} \overline{\mathcal{Q}}_{L} Z q_{R a}$.

(II) Assign the $q_{R}$ 's to the fundamental representation of a new gauge factor $S U(P)$, and $Z$ to the bifundamental $(M, \bar{P})$ of $\mathcal{G}_{M P}$. Then there is a single Yukawa operator $\overline{\mathcal{Q}}_{L} Z q_{R}$.

Note that for $M=N+1$, the two cases coincide; hence, we restrict case (II) to $P \geq 2$. $G_{M N(P)}$ gauge anomalies can be canceled by adding three copies of $M, N,(P)$-plets of colorless "leptons" of chirality opposite to that of the quarks, which can acquire mass from the VEVs of the same multiplets $Y$ and $Z$, e.g., $\sum_{r=1}^{3} \overline{\mathcal{L}_{R}^{r}} Y \ell_{L}^{r}$ etc.

Similarly to $Y$, scalar terms involving only $Z$ also enjoy an exact accidental symmetry $U(1)_{\xi_{Z}}$, i.e., $V(Z)=V\left(Z^{\dagger} Z\right)$. However, by contracting the $S U(M)$ indices of $Y$ and $Z$, we can construct certain mixed non-Hermitian operators that break $U(1)_{\xi_{Y}} \times U(1)_{\xi_{Z}}$ to a single $U(1)$, which is defined by a specific condition relating the two charges $\mathcal{X}_{Y}$ and $\mathcal{X}_{Z}$. As it will become clear below, there are two classes of operators, which we can denote as $\left\{O_{\delta}\right\}$ and $\left\{O_{\epsilon}\right\}$ that correspond to contracting the $S U(M)$ indices with $\delta_{M}=\delta_{\beta}^{\alpha}$ or with $\epsilon_{M}=\epsilon^{\alpha_{1} \ldots \alpha_{M}}$, and which are invariant with respect two different combinations of $U(1)_{\xi_{Y, Z}}$,

$$
\begin{array}{ll}
O_{\delta}: U(1)_{\xi_{Y}} \times U(1)_{\xi_{Z}} \rightarrow U(1)_{\xi}, & \mathcal{X}_{Y}-\mathcal{X}_{Z}=0 \\
O_{\epsilon}: U(1)_{\xi_{Y}} \times U(1)_{\xi_{Z}} \rightarrow U(1)_{\xi^{\prime}}, & N \mathcal{X}_{Y}+P \mathcal{X}_{Z}=0
\end{array}
$$

The charge relation in Eq. (3) implies that $U(1)_{\xi^{\prime}}$ has no QCD anomaly, and hence, the symmetry preserved by

\footnotetext{
${ }^{4}$ Only "square" symmetries $(M=N)$ allow for $U(1)_{\xi_{Y}}$-breaking operators like $\epsilon_{M} \epsilon_{N} Y^{M} \propto \operatorname{det} Y[34,65,66]$.
} 
operators constructed with $\epsilon_{M}$ cannot be promoted to a PQ symmetry. To see this, let us consider a chiral transformation with generic quark charges $\mathcal{X}_{\mathcal{Q}_{L}}, \mathcal{X}_{Q_{R}}, \mathcal{X}_{q_{R}}$. The $U(1)$-QCD anomaly coefficient is, precisely,

$$
|2 \mathcal{N}|=M \mathcal{X}_{\mathcal{Q}_{L}}-N \mathcal{X}_{Q_{R}}-P \mathcal{X}_{q_{R}}=N \mathcal{X}_{Y}+P \mathcal{X}_{Z},
$$

where the relation with the charges of the scalars follows from requiring $U(1)$ invariance of the Yukawa terms.

\section{A. $U(1)$-breaking operators}

Equations (2) and (3) show that operators involving $\delta_{M}$ break $U(1)_{\xi^{\prime}}$, while those involving $\epsilon_{M}$ break $U(1)_{\xi}$. We then need to study which operators can arise, since in the presence of both, no $U(1)$ would survive. Starting with case (I), let us define from the multiplets components $Y_{\alpha i}, Z_{\alpha}$ the set of $S U(N)$ vectors $\left(X_{n}\right)_{i}=\left(Z^{\dagger}\left(Y Y^{\dagger}\right)^{n-1} Y\right)_{i}$, $n=1, \ldots, N$. The operator,

$$
\mathcal{O}_{I}\left(X_{n}\right)=\epsilon_{N} \Pi_{n=1}^{N} X_{n}
$$

does not vanish symmetrically, is nonrenormalizable $(D=N(N+1) \geq 6$ for $N \geq 2)$ and preserves $U(1)_{\xi}$. Since for $M-N \geq 2$ all $\epsilon_{M}$ contractions must involve at least two $Z_{\alpha}$, they vanish symmetrically, and thus, $U(1)_{\xi}$ survives as a perturbatively exact accidental symmetry, broken only by the anomaly with coefficient $|2 \mathcal{N}|=$ $(N+P) \mathcal{X}_{Y}$. A relatively simple example of this case is the $S U(4) \times S U(2)$ model discussed below in Sec. II B. For $M-N=1$, we can instead write

$$
\mathcal{O}_{I}^{\prime}(Y, Z)=(N !)^{-1} \epsilon^{\alpha_{1} \ldots \alpha_{N} \alpha_{M}}\left(\epsilon_{N} Y^{N}\right)_{\alpha_{1} \ldots \alpha_{N}} Z_{\alpha_{M}},
$$

that has dimension $D=M$ (hence is renormalizable for $\mathcal{G}_{32}$ and $\left.\mathcal{G}_{43}\right)$ and preserves $U(1)_{\xi^{\prime}}$. However, $\mathcal{O}_{I}\left(X_{n}\right)$ breaks $U(1)_{\xi^{\prime}}$ at $D=M \cdot N$ so that no $U(1)$ survives.

In case (II), the multiplets components are $Y_{\alpha i}, Z_{\alpha a}$, where $a, b, \ldots$ span $S U(P)$. Let us take $N \geq P \geq 2(N \leq P$ amounts to interchange $Y \leftrightarrow Z)$ and let us consider the $S U(P)$ and $S U(N)$ singlets $\left(\epsilon_{P} Z^{P}\right)_{\alpha_{1} \ldots \alpha_{P}}$ and $\left(\epsilon_{N} Y^{N}\right)_{\beta_{1} \ldots \beta_{N}}$. Since $M=P+N$, the $S U(M)$ indices of their product can be exactly saturated with $\epsilon_{M}$, yielding the $\mathcal{G}_{M N P}$ invariant operator,

$$
\mathcal{O}_{I I}^{\prime}(Y, Z)=(P ! N !)^{-1} \epsilon_{M}\left(\epsilon_{P} Z^{P}\right)\left(\epsilon_{N} Y^{N}\right),
$$

that has a dimension $D=M$ (is renormalizable only for $\mathcal{G}_{422}$ ) and preserves $U(1)_{\xi^{\prime}} \cdot \delta_{M}$ type of operators can be constructed starting from $\left(\epsilon_{P} Z^{\dagger P}\right)^{\alpha_{1} \ldots \alpha_{P}}$ and contracting the $S U(M)$ indices with $P$ components of $Y$. This yields $\left(\epsilon_{P} X_{1}^{P}\right)_{i_{1}, \ldots i_{P}}$, where $\left(X_{1}\right)_{i}^{a}=\left(Z^{\dagger} Y\right)_{i}^{a}$. Only for $N=P$, that is when $X_{1}$ is a $N \times N$ square matrix, we can contract the $S U(N)$ indices with $\epsilon_{N}$. The $D=M$ operator,
TABLE I. Dimension of the operators $\mathcal{O}^{\prime}$ and $\mathcal{O}$ of lowest order that break, respectively, $U(1)_{\xi}$ and $U(1)_{\xi}^{\prime}$. The expression for $D(L)$ is given in the text.

\begin{tabular}{lcc}
\hline \hline Case & $D\left(\mathcal{O}^{\prime}\right)$ & $D(\mathcal{O})$ \\
\hline (I) $M-N=1$ & $M$ & $N(N+1)$ \\
$M-N>1$ & $\cdots$ & $N(N+1)$ \\
(II) $N=P$ & $M$ & $M$ \\
$N>P$ & $M$ & $D(L)$ \\
\hline \hline
\end{tabular}

$$
\mathcal{O}_{I I}\left(X_{1}\right)=(P !)^{-1} \epsilon_{N} \epsilon_{P} X_{1}^{N}=\operatorname{det} X_{1}
$$

(renormalizable only for $\mathcal{G}_{422}$ ) is invariant under $U(1)_{\xi}$. For $P<N \leq 2 P$, new combinations $\left(X_{2}\right)_{i}^{a}=\left(Z^{\dagger} Y Y^{\dagger} Y\right)_{i}^{a}$ allow for the contraction $\left[\epsilon_{N}\left(\epsilon_{P} X_{1}^{P}\right) X_{2}{ }^{N-P}\right]^{a_{1} \ldots a_{N-P}}$. However, unless $N=2 P$, this cannot be contracted into a $P$ singlet. It is then clear that the relevant quantity that we need to consider is the least common multiplier $L \equiv \operatorname{lcm}(P, N)$, in terms of which these operators have the structure,

$$
\mathcal{O}_{I I}\left(X_{n}\right) \sim\left(\epsilon_{N}\right)^{\frac{L}{N}}\left(\epsilon_{P}\right)^{\frac{L}{P}}\left(X_{1}^{P} \ldots X_{\mathcal{F}}^{P} X_{\mathcal{F}+1}^{N-\mathcal{F} P}\right)^{\frac{L}{N}},
$$

where $\mathcal{F} \equiv$ floor $(N / P)$ denotes the greatest integer less or equal to $N / P$. These operators preserve $U(1)_{\xi}$ and break $U(1)_{\xi^{\prime}}$; however, since their dimension $D(L)=$ $(L / N)(\mathcal{F}+1)(2 N-\mathcal{F} P)$ grows rapidly with $L$ (for $N=$ 4 and $P=3, D=30$ ) in most cases $U(1)_{\xi^{\prime}}$ breaking remains an academic issue. In Table I, we list the lowest dimension of operators that break $U(1)_{\xi}$ and $U(1)_{\xi^{\prime}}$.

\section{B. Vacuum structure of the operators}

The PQ solution is endangered when the minimum of the axion potential is shifted away from the one selected by the nonperturbative QCD effects. Therefore, operators that break explicitly $U(1)_{\mathrm{PQ}}$ in the Lagrangian but have vanishing VEVs are harmless, since they do not contribute to determine the minimum. To study the behavior of $\langle\mathcal{O}\rangle$, $\left\langle\mathcal{O}^{\prime}\right\rangle$ at the potential minimum, let us start by considering the renormalizable potential for $Y$. It reads

$$
V(Y)=\kappa\left(T-\mu_{Y}^{2}\right)^{2}+\lambda A,
$$

where $T$ and $A$ are the two invariants introduced above, we require $\kappa>0$ and $\lambda>-\frac{2 N}{N-1} \kappa$ to ensure a potential bounded from below, and $\mu_{Y}^{2}>0$ to trigger SSB. Let us now write $Y(x)$ in its singular value decomposition (SVD),

\footnotetext{
${ }^{5}$ Different field combinations $X_{2} \neq X_{1}$ are needed because otherwise $\left(\epsilon_{N} X_{1}^{N}\right)$ would vanish symmetrically since pairs of $X_{1}$ would necessary have the same $S U(P)$ index. Thus, for $N=m P$, $m$ different objects up to $X_{m}=Z^{\dagger}\left(Y Y^{\dagger}\right)^{m-1} Y$ are needed.
} 


$$
\frac{\sqrt{2}}{v_{Y}} Y=\mathcal{U} \hat{Y} \mathcal{V}^{\dagger}=U \hat{Y} e^{i \varphi_{Y}} V^{\dagger} \rightarrow \hat{Y} e^{i \varphi_{Y}},
$$

where $v_{Y}=\sqrt{2\langle T\rangle}, \mathcal{U}$ and $\mathcal{V}$ are $U(M)$ and $U(N)$ unitary matrices, $U$ and $V$ are the corresponding special matrices with $\operatorname{det}(U, V)=+1, \varphi_{Y}$, defined as

$$
\varphi_{Y}(x) \equiv \frac{a_{Y}(x)}{v_{Y}}=\frac{1}{M} \arg \operatorname{det} \mathcal{U}-\frac{1}{N} \arg \operatorname{det} \mathcal{V},
$$

is the NGB of the global $U(1)_{\xi_{Y}}$, and $\hat{Y}$ is the matrix of real non-negative singular values, which can be taken to lie in the diagonal upper $N \times N$ block with all other entries vanishing. We will henceforth denote by $\left.Y\right|_{N \uparrow}$, the $N \times N$ upper left block of a matrix $Y$. The last form in Eq. (11) is obtained by gauging away $U(x)$ and $V(x)$. In this gauge, the two invariants read

$$
T(\hat{Y})=\sum_{i=1}^{N} y_{i}^{2}, \quad A(\hat{Y})=\sum_{i<j} y_{i}^{2} y_{j}^{2} .
$$

It is now easy to identify the vacuum configurations $\hat{Y}^{c} \equiv\langle\hat{Y}\rangle$ that minimize $V(Y)$ [65]: $T$ is blind to specific orientations of $\hat{Y}$ in field space. This is because it carries a $S O(2 \times M \times N)$ symmetry, which is much larger than $\mathcal{G}_{M N}$, and allows us to rotate different configurations into each other. Adopting the classification of Ref. [67], it is a "flavor irrelevant" operator. The structure of $\hat{Y}^{c}$ is then determined by the extrema of $A(Y)$. Since $A$ is nonnegative, its minimum occurs at $\langle A\rangle=0$, that is when all $y_{i}$ 's but one vanish. The maximum instead occurs at the point of enhanced symmetry $y_{i}^{c}=1 / \sqrt{N}, \forall i$. The sign of $\lambda$ thus determines which minimum is selected. We take $\lambda<$ 0 so that

$$
\left.\hat{Y}^{c}\right|_{N \uparrow}=\operatorname{diag}(1, \ldots, 1) / \sqrt{N} .
$$

The little group is $\mathcal{H}=S U(N)_{V} \times S U(M-N)$ with $S U(N)_{V}$ the "diagonal" combination of $S U(N)$ and of $S U(N)^{\prime} \subset S U(M)$, while the value of $\varphi_{Y}^{c}$ is left undetermined.

With regards to the renormalizable potential for the scalar multiplet $Z$, we need to distinguish between the two cases (I) $Z \sim(M, 1)$ and (II) $Z \sim(M, \bar{P})$. In case (I), the potential has the form Eq. (10) (with $\mu_{Y} \rightarrow \mu_{Z}$ ), but with $A\left(Z_{\alpha}\right)=0$. In the SVD Eq. (11), $V \rightarrow V_{Z}=I$, while $\hat{Z}$ has a single nonzero entry in some row $\alpha$ with $\operatorname{VEV~} z_{\alpha}^{c}=1$.

In case (II), the matrix $V_{Z}$ appearing in the right-hand side of the SVD can be gauged away via a $S U(P)$ transformation, so that we can write

$$
\frac{\sqrt{2}}{v_{Z}} Z \rightarrow U_{Z} \hat{Z} e^{i \varphi_{Z}}
$$

where $\hat{Z}$ has $P$ singular values located in different rows/ columns. For $\lambda_{Z}<0$, the potential is lowered when $\langle A(Z)\rangle$ is maximum, which corresponds to $z_{a}^{c}=1 / \sqrt{P}, \forall a$. The relative orientation of $\langle Y\rangle$ and $\langle Z\rangle$ is determined by the $D=4$ Hermitian operator,

$$
\mathcal{O}_{Z Y}=\operatorname{Tr}\left(Z^{\dagger} Y Y^{\dagger} Z\right) \text {. }
$$

If the coupling is negative, the potential is lowered when $\left\langle\mathcal{O}_{Z Y}\right\rangle$ is maximum. Since $\left.\left\langle Y Y^{\dagger}\right\rangle\right|_{N \uparrow} \propto I_{N \times N}$ (with vanishing entries in the lower $P \times P$ block), this occurs when the $P$ entries $z_{a}$ fall in the upper $N$ positions of $\hat{Z}^{c}$, and $U_{Z}^{c}$ restricted to the block corresponding to these entries is unitary. In short, $\langle Y\rangle$ and $\langle Z\rangle$ get maximally aligned, and in this case, all $\epsilon_{M}$ type of operators $\mathcal{O}^{\prime}$ vanish on the vacuum. If the coupling is positive, then $\left\langle\mathcal{O}_{Z Y}\right\rangle \rightarrow 0$ which is obtained when the entries $z_{a}$ fill the lower $P$ positions of $\hat{Z}^{c}$, and only $\left.U_{Z}^{c}\right|_{P \downarrow} \subset U_{Z}^{c}$ is nontrivial (i.e., with nonvanishing off diagonal entries). $\langle Y\rangle$ and $\langle Z\rangle$ are maximally misaligned, which implies that all operators of $\delta_{M}$ type vanish on the vacuum: $\langle\mathcal{O}\rangle=0 .\left.U_{Z}^{c}\right|_{P \downarrow}$ is unitary but otherwise undetermined. However, in case (II), the $D=M$ operator $\mathcal{O}_{I I}^{\prime}$ Eq. (7) is allowed. The potential is further lowered proportionally to $\propto\left|\left\langle\mathcal{O}_{I I}^{\prime}\right\rangle\right|$, which is maximum for $\left.U_{Z}^{c}\right|_{P \downarrow} \rightarrow I_{P \times P}$.

We should stress at this point that since our study focused on generic gauge invariant combinations of the scalar fields, the results we have obtained hold also in the presence of other sources of PQ breaking, e.g., from operators involving the fermions. In fact, while fermion loops will indeed contribute to the quantum corrections to the scalar effective potential, whatever form the effective potential will acquire, it can only be written in terms of gauge invariant scalar operators which either preserve the PQ symmetry, or vanish on the vacuum.

Another issue is whether the vanishing vacuum value of PQ breaking operators resulting from specific structures of the field VEVs (e.g., alignment or misalignment) could be lifted by perturbative higher order corrections. Let us assume that at the leading order the symmetry breaking pattern is determined by an operator of the type $\left\{O_{\delta}\right\}$, and let us denote the "aligned" VEV configurations as $\langle Y\rangle=$ $Y_{\delta}$ and $\langle Z\rangle=Z_{\delta}$. The gauge group then breaks to $G_{\delta}$ which is the little group of $Y_{\delta}, Z_{\delta}$. Due to alignment, we have $O_{\epsilon}\left(Y_{\delta}, Z_{\delta}\right)=0$ so that at the lowest order $U(1)_{\xi}$ is unbroken in the vacuum. In order to break $U(1)_{\xi}$, higher order effects must then produce $\left\langle O_{\epsilon}\right\rangle \neq 0$, which in turn requires $\langle Y\rangle \neq Y_{\delta}$ and/or $\langle Z\rangle \neq Z_{\delta}$. However, these new VEV configurations will also break further $G_{\delta}$, and it is known that this cannot happen as a result of perturbative effects $[68,69] .^{6}$

\footnotetext{
${ }^{6}$ This conclusion can be evaded only if there are additional massless non-NGB scalar particles in the theory, as is, for example, the case in the Coleman-Weinberg model [70], but not in our model.
} 


\section{A $S U(4) \times S U(2)$ model}

As a concrete application, let us outline a model in which $U(1)_{\mathrm{PQ}}$ arises automatically and is perturbatively exact (details of the phenomenology will be discussed elsewhere). Case (I) with $M-N>1$ is particularly favorable, since $\epsilon_{M}$ operators that could endanger the anomalous $U(1)_{\xi}$ are not allowed (see Table I). The smallest group in this class is $\mathcal{G}_{42}=S U(4) \times S U(2)$. We take $Y \sim(4, \overline{2})$, $Z \sim(4,1), \mathcal{Q}_{L} \sim(4,1), Q_{R} \sim(1,2)$ and $q_{R}^{a}=(1,1)$ (with $a=1,2)$. The flavor relevant scalar terms and the quark Yukawa operators are

$$
\begin{gathered}
V_{f}=-\lambda A(Y)+\eta \mathcal{O}_{Z Y}+\left[\eta_{I} \mathcal{O}_{I}^{(6)}+\text { H.c. }\right], \\
V_{q}=\kappa_{Q} \overline{\mathcal{Q}}_{L} Y Q_{R}+\sum_{a=1,2} \kappa_{a} \overline{\mathcal{Q}}_{L} Z q_{R}^{a}+\text { H.c. }
\end{gathered}
$$

with $\lambda, \eta>0 . \quad A(Y)$ drives $\left.\hat{Y} \rightarrow \hat{Y}^{c}\right|_{2 \uparrow} \sim \operatorname{diag}(1,1)$ at the minimum, while $\mathcal{O}_{Z Y}$ misaligns $\langle Y\rangle$ and $\langle Z\rangle \sim$ $\left(0,0, z_{1}, z_{2}\right)^{T}$, so that $\mathcal{G}_{42}$ breaks to $S U(2)_{V}$. At $D=4$, there are two global symmetries $U(1)_{\xi_{Y}} \times U(1)_{\xi_{Z}}=$ $U(1)_{\xi} \times U(1)_{\xi^{\prime}}$ and, although $\mathcal{O}_{I}^{(6)}$ [see Eq. (5)] breaks $U(1)_{\xi^{\prime}}$ at $D=6$, VEVs misalignment implies $\left\langle\mathcal{O}_{I}^{(6)}\right\rangle=0$ so that there are two NGB,

$a=\frac{1}{v_{a}}\left(v_{Y} a_{Y}+v_{Z} a_{Z}\right), \quad a^{\prime}=\frac{1}{v_{a}}\left(v_{Y} a_{Y}-v_{Z} a_{Z}\right)$,

where $v_{a}^{2}=v_{Y}^{2}+v_{Z}^{2}$ and, given that all the fields have the same periodicity, we have set $\mathcal{X}_{Y}=\mathcal{X}_{Z}=1 . a(x)$ gets a mass $m_{a} \sim m_{\pi} f_{\pi} / f_{a}$ from the QCD anomaly with $f_{a}=$ $v_{a} /|2 \mathcal{N}|$ and $|2 \mathcal{N}|=2\left(\mathcal{X}_{Y}+\mathcal{X}_{Z}\right)=4$. There are, however, only two domain walls because under the $\mathbb{Z}_{2}$ center of $S U(2)_{V}\langle a\rangle \rightarrow\langle a\rangle+\pi$. At this stage, $a^{\prime}(x)$ remains massless. However, considering that breaking $U(1)_{\xi^{\prime}}$ does not imply breaking the gauge symmetry, $a^{\prime}$ might eventually acquire a mass à la Coleman-Weinberg [70] once all the effects, including those of the fermions, are included in the effective potential.

\section{A GAUGE SYMMETRY FOR A CLOCKWORK AXION}

We now discuss a construction based on rectangular gauge symmetries that enforces a mechanism for a highly protected "clockwork" $\tilde{U}(1)_{\mathrm{PQ}}$. While this the model embeds some of the properties of the semisimple rectangular gauge groups discussed above, it differs from the previous constructions in that the local gauge group includes an Abelian factor, whose role is crucial to enforce the accidental symmetry. Note also that although we use suggestive names for some group factors, this should be regarded as a toy model, not intended to describe real phenomenology.
Consider the gauge group $U(1)_{y} \times[S U(2) \times S U(3)]^{n+1}$. We call $U(1)_{\mathcal{Y}}$ hyperchage, and the first $S U(2) \times S U(3)$ non-Abelian factors isospin and flavor. We introduce three sets of quarks in the fundamental of color transforming under these factors as $Q_{L} \sim(2,3)_{\frac{1}{3}}, u_{R}^{a} \sim(1,1)_{\frac{4}{3}}, d_{R}^{a} \sim$ $(1,1)_{-\frac{2}{3}}$ with $a=1,2,3$ and two scalar multiplets $Y_{d, u} \sim$ $(2,3)_{ \pm 1}$ acquiring VEVs $\left\langle T\left(Y_{d, u}\right)\right\rangle=v_{d, u}^{2} / 2$. (We leave understood that gauge anomalies can be compensated by a suitable set of "leptons.") The Yukawa Lagrangian reads

$$
\mathcal{L}_{q}=-\sum_{a=1}^{3}\left(\kappa_{u}^{a} \bar{Q}_{L} Y_{u} u_{R}^{a}+\kappa_{d}^{a} \bar{Q}_{L} Y_{d} d_{R}^{a}\right)+\text { H.c. }
$$

where $\kappa_{u, d}^{a}$ are coupling constants. Note that a coupling $\left(\epsilon_{2} Y_{u} Y_{d}\right)_{\alpha \beta}$ is forbidden because of unsaturated flavor indices, so that the scalar potential involving $Y_{u, d}$ has the form $V\left(Y_{u}^{\dagger} Y_{u}, Y_{d}^{\dagger} Y_{d}\right)$ and, besides local $U(1)_{\mathcal{Y}}$, it carries also an additional accidental symmetry, that is $U(1)_{\xi_{u}} \times U(1)_{\xi_{d}}=U(1)_{\mathcal{Y}} \times U(1)_{\xi}$. Orthogonality with hypercharge,

$$
\mathcal{Y}_{u} \mathcal{X}_{u} v_{u}^{2}+\mathcal{Y}_{d} \mathcal{X}_{d} v_{d}^{2}=0
$$

fixes the ratio of the $U(1)_{\xi}$ charges of the scalars as $\mathcal{X}_{u} / \mathcal{X}_{d}=v_{d}^{2} / v_{u}^{2}$, and we normalize their sum to $\mathcal{X}_{u}+\mathcal{X}_{d}=2$. We now add two sets of hyperchargeless fields $\Sigma_{p}, Y_{p}(p=1, \ldots, n)$ that transform under the additional gauge factors. For the first three non-Abelian group factors in the chain, that is $S U(3) \times S U(2)_{1} \times$ $S U(3)_{1}$, we have

$$
\Sigma_{1 \alpha}^{\alpha_{1} i_{1}} \sim\left(3, \overline{2}_{1}, \overline{3}_{1}\right), \quad Y_{1 \alpha_{1} i_{1}} \sim\left(1,2_{1}, 3_{1}\right) \ldots,
$$

with the successive factors $p>1 \Sigma_{p} \sim\left(3_{p-1}, \overline{2}_{p}, \overline{3}_{p}\right)$ and $Y_{p} \sim\left(1,2_{p}, 3_{p}\right)$. This allows us to write a chain of $n$ renormalizable operators,

$$
\left(\epsilon_{3} \epsilon_{2} Y_{u} Y_{d} \Sigma_{1}\right)^{\alpha_{1} i_{1}} Y_{1 \alpha_{1} i_{1}}+\sum_{p=2}^{n}\left(\epsilon_{3} \epsilon_{2} Y_{p-1}^{2} \Sigma_{p}\right)^{\alpha_{p} i_{p}} Y_{p_{\alpha_{p} i_{p}}}
$$

For each field $\Sigma_{p}$, we can write a $D=6$ operator $\epsilon_{2}^{3}\left(\epsilon_{3} \epsilon_{3^{\prime}} \Sigma_{p}^{3}\right)\left(\epsilon_{3} \epsilon_{3^{\prime}} \Sigma_{p}^{3}\right)$ which, together with the operators in Eq. (22), breaks the global symmetry $U(1)_{\xi} \times$ $\left[U(1)_{\Sigma} \times U(1)_{Y}\right]^{n}$ to $\tilde{U}(1)_{\mathrm{PQ}}$ under which $\tilde{\mathcal{X}}_{Y_{1}}=-\left(\tilde{\mathcal{X}}_{u}+\right.$ $\left.\tilde{\mathcal{X}}_{d}\right)=-2$ and in general, $\tilde{\mathcal{X}}_{Y_{p}}=(-2)^{p}$ while $\tilde{\mathcal{X}}_{\Sigma}=0$

Let us now assume that there is no large scale in the model, and that all dimensional parameters in the scalar potential are of order $v_{u, d}$. The operators in Eq. (22) have multifold effects. First, nonvanishing VEVs would lower the potential by an amount $\sim\left|\left\langle Y Y \Sigma^{\prime} Y^{\prime}\right\rangle\right|$; hence, the field 
VEVs tend to align in specific directions. The combination $\epsilon_{2} Y_{u} Y_{d}$ in the first term misaligns $\left\langle Y_{u}\right\rangle$ and $\left\langle Y_{d}\right\rangle$ in isospin space, in such a way that upon $U(1)_{\mathcal{Y}} \times S U(2)$ breaking a $U(1)$ gauge factor is preserved in the usual way. At the same time, $\epsilon_{3} Y_{u} Y_{d} \Sigma_{1}$ rotates $\left\langle\Sigma_{1}\right\rangle$ in flavor space in the direction orthogonal to $\left\langle Y_{u}\right\rangle$ and $\left\langle Y_{d}\right\rangle$ while, because of $\delta_{2_{1}}$ and $\delta_{3_{1}}$ index contraction, $\left\langle\Sigma_{1}\right\rangle$ and $\left\langle Y_{1}\right\rangle$ get aligned in $S U(3)_{1} \times S U(2)_{1}$ space. Isospin breaking provides a negative mixed term $-v_{u} v_{d} \Sigma_{1} Y_{1}$ so that there are regions in parameter space where $\Sigma_{1}$ and $Y_{1}$ acquire VEVs proportional to $v_{u, d}$ even if their mass square terms are nonnegative. By extrapolation, regions exist in which all the VEVs in the chain vanish if isospin is unbroken.

Let us now study if $\tilde{U}(1)_{\mathrm{PQ}}$ can be broken by higher dimension operators. At $D=6$, we can write

$$
\epsilon_{i j}\left(Y_{1}^{\dagger} \Sigma_{1}^{\dagger} Y_{u}\right)_{i}\left(Y_{1}^{\dagger} \Sigma_{1}^{\dagger} Y_{d}\right)_{j}
$$

This operator vanishes on the vacuum because the first term in Eq. (22) rotates $\left\langle\Sigma_{1}\right\rangle$ in the direction orthogonal in flavor space to $\left\langle Y_{u}\right\rangle$ and $\left\langle Y_{d}\right\rangle$. As regards the other multiplets, for each pair $\left(\Sigma_{p+1}, Y_{p+1}\right)$, let us now define $\left(X_{n}\right)_{\gamma_{p}}=\left[\Sigma\left(\Sigma^{\dagger} \Sigma\right)^{n-1} Y\right]_{\gamma_{p}}$ with $n \geq 1$, in terms of which we can write the following two operators:

$$
\epsilon_{2_{p}}\left(X_{1}^{\dagger} Y_{p}\right)\left(X_{2}^{\dagger} Y_{p}\right)(D=8), \quad \epsilon_{3_{p}} X_{1} X_{2} X_{3}(D=12),
$$

which also break $\tilde{U}(1)_{\mathrm{PQ}}$. However, all $\left\langle X_{n}\right\rangle$ 's have a single nonzero component in $S U(3)_{p}$ space, orthogonal to the plane $\left\langle Y_{p}\right\rangle_{\alpha_{p}}-\left\langle Y_{p}\right\rangle_{\beta_{p}}$, and this ensures that also these $\tilde{U}(1)_{\mathrm{PQ}}$ breaking operators vanish on the vacuum. Thus, $\tilde{U}(1)_{\mathrm{PQ}}$ remains perturbatively exact, broken only by the QCD anomaly with coefficient $|2 \mathcal{N}|=3\left(\mathcal{X}_{u}+\mathcal{X}_{d}\right)=6$. The corresponding NGB is

$$
\tilde{a}(x)=\frac{1}{v_{a}}\left(v_{u} a_{u}+v_{d} a_{d}+\sum_{p=1}^{n} v_{p} a_{p}\right),
$$

where $v_{u, d, p}$ and $a_{u, d, p}$ are the VEVs and orbital modes of $Y_{u, d, p}$, and

$$
v_{a}^{2}=\tilde{\mathcal{X}}_{u}^{2} v_{u}^{2}+\tilde{\mathcal{X}}_{d}^{2} v_{d}^{2}+\sum_{p} \tilde{\mathcal{X}}_{p}^{2} v_{p}^{2} \approx \frac{v^{2}}{3} 4^{n+1},
$$

where the approximation holds for $v_{u, d, p} \approx v$. Taking now the isospin (and PQ) breaking VEVs at a scale $v \sim 100 \mathrm{GeV}$, for $n \sim 20$, the radius of the axion compact space is boosted to $v_{a} \gtrsim 10^{8} \mathrm{GeV}$ without introducing any large fundamental scale.

\section{CONCLUSIONS}

The "origin" and "quality" problems of the PQ symmetry can be solved by assigning the scalar multiplets hosting the axion to representations of semisimple gauge groups with a "rectangular" structure. No group factors of large degree are required, which renders this solution particularly elegant. It should have not gone unnoticed that such constructions require that (exotic) quarks must replicate, with some "generations" obtaining a mass from different VEVs than others. Admittedly, embedding rectangular symmetries in the SM to play the role of flavor symmetries appears as a challenging undertaking, but hopefully not insurmountable. Succeeding in this venture might uncover unexpected implications for the SM flavor problem.

\section{ACKNOWLEDGMENTS}

We acknowledge conversations with G. Grilli di Cortona. The authors are supported by the INFN Iniziativa Specifica, Theoretical Astroparticle Physics (TAsP-LNF).
[1] A. A. Belavin, A. M. Polyakov, A. S. Schwartz, and Yu. S. Tyupkin, Pseudoparticle solutions of the Yang-Mills equations, Phys. Lett. 59B, 85 (1975).

[2] C. G. Callan, Jr., R. F. Dashen, and D. J. Gross, The structure of the gauge theory vacuum, Phys. Lett. 63B, 334 (1976).

[3] R. Jackiw and C. Rebbi, Vacuum Periodicity in a YangMills Quantum Theory, Phys. Rev. Lett. 37, 172 (1976).

[4] L. Ubaldi, Effects of theta on the deuteron binding energy and the triple-alpha process, Phys. Rev. D 81, 025011 (2010).

[5] M. Dine, L.S. Haskins, L. Ubaldi, and D. Xu, Some remarks on anthropic approaches to the strong $C P$ problem, J. High Energy Phys. 05 (2018) 171.
[6] D. Lee, U.-G. Meißner, K. A. Olive, M. Shifman, and T. Vonk, $\theta$-dependence of light nuclei and nucleosynthesis, Phys. Rev. Research 2, 033392 (2020).

[7] R. D. Peccei and H. R. Quinn, $C P$ Conservation in the Presence of Instantons, Phys. Rev. Lett. 38, 1440 (1977).

[8] R. D. Peccei and H. R. Quinn, Constraints imposed by $C P$ conservation in the presence of instantons, Phys. Rev. D 16, 1791 (1977).

[9] S. Weinberg, A New Light Boson?, Phys. Rev. Lett. 40, 223 (1978).

[10] F. Wilczek, Problem of Strong $p$ and $t$ Invariance in the Presence of Instantons, Phys. Rev. Lett. 40, 279 (1978).

[11] L. F. Abbott and P. Sikivie, A cosmological bound on the invisible axion, Phys. Lett. 120B, 133 (1983). 
[12] M. Dine and W. Fischler, The not so harmless axion, Phys. Lett. 120B, 137 (1983).

[13] J. Preskill, M. B. Wise, and F. Wilczek, Cosmology of the invisible axion, Phys. Lett. 120B, 127 (1983).

[14] L. Di Luzio, M. Giannotti, E. Nardi, and L. Visinelli, The landscape of QCD axion models, Phys. Rep. 870, 1 (2020).

[15] M. Dine and N. Seiberg, String theory and the strong $C P$ problem, Nucl. Phys. B273, 109 (1986).

[16] S. M. Barr and D. Seckel, Planck scale corrections to axion models, Phys. Rev. D 46, 539 (1992).

[17] M. Kamionkowski and J. March-Russell, Planck scale physics and the Peccei-Quinn mechanism, Phys. Lett. B 282, 137 (1992).

[18] R. Holman, S. D. H. Hsu, T. W. Kephart, E. W. Kolb, R. Watkins, and L. M. Widrow, Solutions to the strong $C P$ problem in a world with gravity, Phys. Lett. B 282, 132 (1992).

[19] S. Ghigna, M. Lusignoli, and M. Roncadelli, Instability of the invisible axion, Phys. Lett. B 283, 278 (1992).

[20] L. Randall, Composite axion models and Planck scale physics, Phys. Lett. B 284, 77 (1992).

[21] L.F. Abbott and M.B. Wise, Wormholes and global symmetries, Nucl. Phys. B325, 687 (1989).

[22] S. R. Coleman and K.-M. Lee, Wormholes made without massless matter fields, Nucl. Phys. B329, 387 (1990).

[23] R. Kallosh, A. D. Linde, D. A. Linde, and L. Susskind, Gravity and global symmetries, Phys. Rev. D 52, 912 (1995).

[24] R. Alonso and A. Urbano, Wormholes and masses for Goldstone bosons, J. High Energy Phys. 02 (2019) 136.

[25] J. Alvey and M. Escudero, The axion quality problem: Global symmetry breaking and wormholes, J. High Energy Phys. 01 (2021) 032.

[26] L. M. Krauss and F. Wilczek, Discrete Gauge Symmetry in Continuum Theories, Phys. Rev. Lett. 62, 1221 (1989).

[27] E. J. Chun and A. Lukas, Discrete gauge symmetries in axionic extensions of the SSM, Phys. Lett. B 297, 298 (1992).

[28] A. G. Dias, V. Pleitez, and M. D. Tonasse, Naturally light invisible axion in models with large local discrete symmetries, Phys. Rev. D 67, 095008 (2003).

[29] L. M. Carpenter, M. Dine, and G. Festuccia, Dynamics of the Peccei Quinn scale, Phys. Rev. D 80, 125017 (2009).

[30] K. Harigaya, M. Ibe, K. Schmitz, and T. T. Yanagida, Peccei-Quinn symmetry from a gauged discrete R symmetry, Phys. Rev. D 88, 075022 (2013).

[31] A. G. Dias, A. C. B. Machado, C. C. Nishi, A. Ringwald, and P. Vaudrevange, The quest for an intermediate-scale accidental axion and further ALPs, J. High Energy Phys. 06 (2014) 037.

[32] K. Harigaya, M. Ibe, K. Schmitz, and T. T. Yanagida, Peccei-Quinn symmetry from dynamical supersymmetry breaking, Phys. Rev. D 92, 075003 (2015).

[33] A. Ringwald and K. Saikawa, Axion dark matter in the post-inflationary Peccei-Quinn symmetry breaking scenario, Phys. Rev. D 93, 085031 (2016); 94, 049908(A) (2016).

[34] L. Di Luzio, E. Nardi, and L. Ubaldi, Accidental PecceiQuinn Symmetry Protected to Arbitrary Order, Phys. Rev. Lett. 119, 011801 (2017).
[35] M. Ardu, L. Di Luzio, G. Landini, A. Strumia, D. Teresi, and J.-W. Wang, Axion quality from the (anti)symmetric of SU $(\mathcal{N})$, J. High Energy Phys. 11 (2020) 090.

[36] H.-S. Lee and W. Yin, Peccei-Quinn symmetry from a hidden gauge group structure, Phys. Rev. D 99, 015041 (2019).

[37] W. Yin, Scale and quality of Peccei-Quinn symmetry and weak gravity conjectures, J. High Energy Phys. 10 (2020) 032 .

[38] B. Lillard and T. M. P. Tait, A composite axion from a supersymmetric product group, J. High Energy Phys. 11 (2017) 005.

[39] B. Lillard and T. M. P. Tait, A high quality composite axion, J. High Energy Phys. 11 (2018) 199.

[40] Y. Nakai and M. Suzuki, Axion quality from superconformal dynamics, Phys. Lett. B 816, 136239 (2021).

[41] C. T. Hill and A. K. Leibovich, Deconstructing 5-D QED, Phys. Rev. D 66, 016006 (2002).

[42] C. T. Hill and A. K. Leibovich, Natural theories of ultralow mass PNGB's: Axions and quintessence, Phys. Rev. D 66, 075010 (2002).

[43] M. Redi and R. Sato, Composite accidental axions, J. High Energy Phys. 05 (2016) 104.

[44] M. Yamada and T. T. Yanagida, A natural and simple UV completion of the QCD axion model, Phys. Lett. B 816, 136267 (2021).

[45] K. Choi and S. H. Im, Realizing the relaxion from multiple axions and its UV completion with high scale supersymmetry, J. High Energy Phys. 01 (2016) 149.

[46] D. E. Kaplan and R. Rattazzi, Large field excursions and approximate discrete symmetries from a clockwork axion, Phys. Rev. D 93, 085007 (2016).

[47] T. Higaki, K. Sik Jeong, N. Kitajima, and F. Takahashi, The QCD axion from aligned axions and diphoton excess, Phys. Lett. B 755, 13 (2016).

[48] G. F. Giudice and M. McCullough, A clockwork theory, J. High Energy Phys. 02 (2017) 036.

[49] A. Ahmed and B. M. Dillon, Clockwork goldstone bosons, Phys. Rev. D 96, 115031 (2017).

[50] N. Craig, I. G. Garcia, and D. Sutherland, Disassembling the clockwork mechanism, J. High Energy Phys. 10 (2017) 018.

[51] G. F. Giudice and M. McCullough, Comment on "Disassembling the clockwork mechanism", arXiv:1705.10162.

[52] K. Choi, S. H. Im, and C. S. Shin, General continuum clockwork, J. High Energy Phys. 07 (2018) 113.

[53] G. F. Giudice, Y. Kats, M. McCullough, R. Torre, and A. Urbano, Clockwork/linear dilaton: Structure and phenomenology, J. High Energy Phys. 06 (2018) 009.

[54] D. Teresi, Clockwork without supersymmetry, Phys. Lett. B 783, 1 (2018).

[55] M. Farina, D. Pappadopulo, F. Rompineve, and A. Tesi, The photo-philic QCD axion, J. High Energy Phys. 01 (2017) 095.

[56] L. Di Luzio, F. Mescia, and E. Nardi, Window for preferred axion models, Phys. Rev. D 96, 075003 (2017).

[57] L. Darmé, L. Di Luzio, M. Giannotti, and E. Nardi, Selective enhancement of the QCD axion couplings, Phys. Rev. D 103, 015034 (2021).

[58] K. Choi, S. H. Im, and C. S. Shin, Recent progresses in physics of axions or axion-like particles, arXiv:2012.05029. 
[59] T. Higaki, K. S. Jeong, N. Kitajima, and F. Takahashi, Quality of the Peccei-Quinn symmetry in the aligned QCD axion and cosmological implications, J. High Energy Phys. 06 (2016) 150.

[60] R. Coy, M. Frigerio, and M. Ibe, Dynamical clockwork axions, J. High Energy Phys. 10 (2017) 002.

[61] A. J. Long, Cosmological aspects of the clockwork axion, J. High Energy Phys. 07 (2018) 066.

[62] Q. Bonnefoy, E. Dudas, and S. Pokorski, Axions in a highly protected gauge symmetry model, Eur. Phys. J. C 79, 31 (2019).

[63] H. Georgi and A. Pais, Vacuum symmetry and the pseudoGoldstone phenomenon, Phys. Rev. D 12, 508 (1975).

[64] J. R. Espinosa, C. S. Fong, and E. Nardi, Yukawa hierarchies from spontaneous breaking of the $S U(3)_{L} \times S U(3)_{R}$ flavour symmetry?, J. High Energy Phys. 02 (2013) 137.
[65] E. Nardi, Naturally large Yukawa hierarchies, Phys. Rev. D 84, 036008 (2011).

[66] C. S. Fong and E. Nardi, Spontaneous Breaking of Flavor Symmetry Avoids the Strong CP Problem, Phys. Rev. Lett. 111, 061601 (2013).

[67] C. S. Fong and E. Nardi, Quark masses, mixings, and $C P$ violation from spontaneous breaking of flavor $S U(3)^{3}$, Phys. Rev. D 89, 036008 (2014).

[68] H. Georgi and A. Pais, $C P$-violation as a quantum effect, Phys. Rev. D 10, 1246 (1974).

[69] H. Georgi and A. Pais, Natural stepwise breaking of gauge and discrete symmetries, Phys. Rev. D 16, 3520 (1977).

[70] S. R. Coleman and E. J. Weinberg, Radiative corrections as the origin of spontaneous symmetry breaking, Phys. Rev. D 7, 1888 (1973). 